\title{
Lien, sens et action : vers une communication engageante
}

Françoise Bernard et Robert Vincent Joule

\section{(2) OpenEdition}

12 Journals

Édition électronique

URL : http://journals.openedition.org/communicationorganisation/2918

DOI : 10.4000/communicationorganisation.2918

ISSN : $1775-3546$

Éditeur

Presses universitaires de Bordeaux

Édition imprimée

Date de publication : 1 mai 2004

ISSN : 1168-5549

Référence électronique

Françoise Bernard et Robert Vincent Joule, « Lien, sens et action : vers une communication engageante », Communication et organisation [En ligne], 24 | 2004, mis en ligne le 27 mars 2012, consulté le 19 avril 2019. URL : http://journals.openedition.org/communicationorganisation/2918 DOI : 10.4000/communicationorganisation.2918

Ce document a été généré automatiquement le 19 avril 2019

(c) Presses universitaires de Bordeaux 


\title{
Lien, sens et action : vers une communication engageante
}

\author{
Françoise Bernard et Robert Vincent Joule
}

\section{Introduction}

1 Le projet qui est le nôtre est de souligner les liens entre le co exister et le co agir.

2 Nous aborderons le co exister sous l'angle du co agir en considérant. dans le travail que nous présentons, que dans les organisations, l'exister ensemble peut être observé comme effet de l'agir ensemble.

3 La réflexion présentée repose, d'une part, sur une expérience d'approche et de coopération pluridisciplinaire et d'autre part, sur une étude empirique issue d'un projet de changement conduit dans une organisation.

4 En ce qui concerne l'étude empirique au sens strict nous présentons les résultats d'une intervention d'une année comportant la conception et le guidage d'un projet de changement centré sur le développement de «comportements sécurité ». Cette intervention a eu lieu dans une grande entreprise du secteur public: le Groupe Charbonnages de France, sur fond de cessation d'activités. Cette étude prend appui, en ce qui concerne sa dimension théorique, sur une construction pluridisciplinaire, en l'occurrence un croisement entre théorie de l'engagement (psychologie sociale) et théorie de lu communication des organisations. Les choix théoriques ont été laits dans une perspective heuristique.

5 En ce qui concerne l'expérience d'approche et de coopération pluridisciplinaire, ce projet s'inscrit dans un "chantier» de recherche pluridisciplinaire plus large consacré à la construction du paradigme de la «communication engageante» impliquant des chercheurs en sciences de l'information et de la communication et en psychologie sociale, $\mathrm{Lu}$ tel chantier s'inscrit dans une perspective à long terme visant à articuler les problématiques du sens, du lien et de l'action. 
6 Dans le cadre ainsi défini, nous présenterons, dans un premier temps, brièvement, l'approche empirique puis, nous privilégierons une présentation de la théorie de l'engagement, qui est associée dans cette étude à la théorie de la communication de projet 1. Enfin, nous présenterons certains éléments constitutifs du paradigme de la « communication engageante » dans l'esprit d'un bilan d'étape.

\section{Présentation de l'approche empirique}

7 Le choix de l'étude empirique présentée est donc une contribution au paradigme de la «communication engageante» d'une part et d'autre part, illustre le lien que nous proposons d'établir entre le co agir et le co exister. En ce qui concerne ce dernier point, le projet conduit dans l'organisation qui constitue notre objet d'étude l'a été à un moment d'essoufflement et d'affaiblissement de la culture et du lien de travail et au bout du compte du "travailler ensemble» et de l'«exister ensemble» comme nous allons l'exposer. Ce projet fortement marqué par des logiques d'action a permis de relancer une forme du « co exister » dans les équipes de travail, entre les équipes de travail et entre les niveaux hiérarchiques.

8 L'Unité d'Exploitation de Provence (UE) des Houillères de Bassin du Centre et du Midi du Groupe Charbonnages de France est issue de la fusion de trois sociétés en 1946 dans le cadre de la loi de nationalisation ${ }^{2}$ Cette année là l'effectif salarié était de 6182 personnes et la production d'un million détonnes : en 2001. 324001 tonnes avaient été produites avec 560 agents sur un seul chantier entièrement mécanisé (le gisement étant situé à plus de 1300 mètres de profondeur). La production avait été fixée à un seuil de 200000 tonnes jusqu'à la fermeture en janvier 2003. La lignite provençale produite est destinée à la production d'électricité dans la centrale thermique de Provence (Société Nationale d'Electricité et de Thermique SNLI) filiale du Groupe Charbonnages de France. La dernière taille. « la taille 24 » a eu été exploitée en 2002 avec le double enjeu de montrer jusqu’à la tin le professionnalisme de l'UE de Provence cl de veiller strictement à la sécurité du personnel.

9 Les enjeux et pratiques de sécurité sont stratégiques et centraux pour le Groupe Charbonnages de France. La perspective de l'arrêt programmé des activités a conduit la direction nationale à définir la sécurité comme objectif prioritaire depuis 1995. Les enjeux financiers sont à la hauteur de cette ambition, à titre d'exemple, en 2000 près de $11 \mathrm{M}$ d'euros avaient été engagés à l'aménagement d'équipements et $2.5 \mathrm{M}$ d'euros à la recherche pour la sécurité collective (aérage. grisou, pression de terrain, explosion) ${ }^{3}$.

10 La sécurité, enjeu prioritaire ${ }^{4}$, s'inscrit historiquement dans des programmes, conçus et conduits par les services sécurité, fortement marqués par une approche technique et réglementaire. Cependant, en complément des enjeux de sécurité collective, la question du comportement individuel de sécurité a émergé au fil du temps et est devenue un centre d'intérêt clairement affirmé notamment à partir de 1996 où il s'est agi de poursuivre jusqu'au bout l'exploitation (2005 au niveau national, début 2003 au niveau régional) en garantissant l'intégrité physique de chacun.

11 C'est dans un tel contexte "d'amélioration du comportement sécurité » que le projet présenté a été conduit.

12 Ce projet peut être défini de la manière suivante: afin de travailler la question du comportement sécurité dans une perspective heuristique et dans le but d'obtenir des 
résultats concrets, il a semblé pertinent de croiser des connaissances et des pratiques issues d'une part de la communication des organisations et d'autre part de la psychologie sociale et plus précisément de la psychologie de l'engagement. La problématique étant de conduire les individus dans leurs collectifs de travail à mener des actions concrètes pour préserver leur sécurité personnelle et celle de leurs partenaires de travail.

Ce sont ces actions, à l'intersection permanente entre l'individuel et le collectif, que nous posons comme action critique d'un "exister ensemble». Le présupposé étant qu'en matière de co existence, les actes sont au moins aussi importants que les discours et les représentations.

Par ailleurs et plus largement, notre perspective est de situer les problématiques communicationnelles au carrefour des interactions entre les enjeux et pratiques relatifs au sens, au lien et à l'action. Dans un tel cadre, notre projet est de fonder un socle théorique, méthodologique et pratique pour « une communication engageante ».

\section{Retour sur la théorie de l'engagement}

Formuler la question de la sécurité comme problématiques croisées de communication, d'action et de changement nous a conduit à convoquer la théorie de l'engagement (Joule et Beauvois. IW8. 2002). I,'hypothèse centrale est que le changement, en l'occurrence «tout mettre en œuvre individuellement et collectivement à chaque instant pour garantir la meilleure sécurité possible » suppose d'obtenir des actes concrets de chacun des acteurs. Dans la mesure où ces actes nécessitent dans un ensemble de cas un changement comportemental, l'enjeu est bien de créer les conditions favorables pour que les acteurs s'engagent dans un cours d'action, la notion de "conditions favorables" renvoie aux propositions théoriques de la psychologie tic l'engagement d'une part et d'autre part à des propositions de communication.

la communication, centrée sur les processus socio-sémiotiques et discursifs, est susceptible de taire évoluer les attitudes, croyances et opinions, comme on le sait, des idées aux actes, il peut $\mathrm{v}$ avoir un décalage, voire, dans certains cas un gouffre. Or en matière de sécurité ce sont bien les actes et même souvent les micro actes qui comptent car ils peuvent être à l'origine d'accidents ou au contraire de la prévention des accidents.

Aussi, dans un premier temps et dans le but de réduire un tel décalage, nous avons fait appel aux recherches en psychologie sociale qui étudient un tel décalage entre les idées et les actes (voir notamment Joule. 1889 : Channouf. Py et Somat. 1996). Ces travaux, pris dans leur ensemble, nous invitent à nous interroger sur les conditions d'optimalité des campagnes de communication, d'information ou de sensibilisation, pour peu qu'elles ambitionnent de toucher les comportements effectifs. Ces campagnes reposent, pour la plupart, sur le présupposé suivant : les comportements découlant logiquement des idées, il suffit de changer les idées pour changer les comportements : cl pour changer les idées, on table sur les vertus de l'information et de la communication. Or les choses ne sont pas si simples. Les spécialistes de la communication savent que bien souvent, les campagnes d'information et communication n'ont pas l'effet souhaité, cela est vérifié dans de nombreux domaines : la santé, la prévention routière, le civisme, la lutte contre l'échec scolaire, etc.

Dans un deuxième temps, au sein de ces travaux, nous nous sommes intéressés plus spécifiquement au paradigme du " pied-dans-la-porte $»^{5}$. Celui-ci propose une toute autre 
approche, par les actes, qui consiste à produire préalablement un «soubassement comportemental» reformulé en «soubassement comportemental engageant» (Joule 2000) qui est un comportement dit " préparatoire ».

19 Cette notion intéresse directement les spécialistes de la communication, notamment parce que les recherches consacrées au "pied-dans-la-porte» par exemple, nous montrent qu'on a plus de chances d'être entendu lorsque les arguments que l'on avance (ou les informations que l'on donne) ont été précédés par l'obtention d'un comportement préparatoire.

Des auteurs (Burger. 1999) ont défini les principales caractéristiques du contexte dans lequel ce comportement préparatoire doit être obtenu :

1/ le comportement préparatoire doit être effectivement réalisé (il ne faut donc pas se contenter d'intentions comportementales) :

2/ il est bon d'aider la personne à établir un lien entre ce qu'elle vient défaire et ce qu'elle est en recourant à un étiquetage (attribution interne) :

3/ le comportement préparatoire doit avoir un certain coût :

24 4/ le comportement préparatoire et le comportement taisant l'objet de la requête finale doivent relever de la même identification de l'action :

5/ il vaut mieux que ce ne soit pas la même personne qui sollicite le comportement préparatoire et qui formule la requête finale :

6/ le comportement préparatoire ne doit pas être lié à une compensation financière, et de façon plus générale, à quelque promesse de récompense.

27 Ce comportement préparatoire, peu coûteux, dont l'obtention précède l'obtention d'autres comportements plus coûteux non seulement déplace la question de la communication mais propose un cadre d'action communicationnelle que nous avons fait fonctionner dans l'étude présentée.

En ce qui concerne le déplacement de la communication, la question devient comment communiquer un message qui passe par la traduction cognitive d'un soubassement comportemental engageant, puisque cette lois ce qui est dit va pouvoir faire écho avec ce que la personne a pu préalablement faire. En ce qui concerne le cadre d'action communicationnelle il s'agit dépenser en permanence les liens entre communication et action, en l'occurrence action sécurité, en veillant notamment à la cohérence des contenus et des formes avec le cadre théorique de rengagement. Ce dernier point, donne lieu à des décisions pratiques, par exemple : qui rédige le texte de l'affiche "présentation des actions sécurité par équipe ». où convient-il d'exposer cette affiche, etc. Les décisions sont donc prises selon un critère d'engagement par les actes des agents, cl avec l'objectif de plus de sécurité effective.

29 Au-delà de cet apport, nous avons emprunté à la théorie de la psychologie de l'engagement un ensemble d'autres propositions pour conduire notre projet. Pour présenter correctement ces propositions, nous faisons un retour sur les définitions principales.

30 La première définition de l'engagement date du milieu des années soixante. On la doit à kiesler et Sakumura (1966) :

31 "l'engagement est le lien unit l'individu à ses actes comportementaux». 

eux en évidence la double caractéristique de cause et d'effet de la notion d'engagement. C'est précisément parce qu'il $\boldsymbol{y}$ a engagement qu'un lien va pouvoir s'établir entre un individu et ses actes. Et cet engagement est tributaire de la situation, puisque c'est elle en fonction de ses caractéristiques objectives - qui engage ou qui n'engage pas l'individu dans ses actes et qui par voie de conséquence, favorise ou au contraire entrave, voire interdit, l'établissement d'un lien entre lui et ses agissements. lait ce que l'on a fait ou de faire ce que l'on s'apprête à faire. Ces raisons sont d'ordre interne et externe. Les raisons d'ordre interne sont plus engageantes que les raisons d'ordre externe, puisque l'individu en appelle à ce qu'il est pour interpréter sa conduite. Et les « auto attributions », (« c'est dans mu nature de... ») sont d'autant plus fortes que le contexte dans lequel l'individu évolue lui confère un statut de sujet «libre». Pour résumer, il convient donc de mettre en place les conditions qui permettront aux agents de prendre librement la décision de veiller à leur sécurité en trouvant de bonnes raisons «internes » de le faire, par exemple : «je fais attention à la vitesse des engins dans les voies parce que je suis un bon collègue », «je nettoie le chantier parce que je suis un bon mineur ». «je porte mes lunettes parce que j'ai de bons veux et que j'entends les garder ». etc ${ }^{6}$

\section{Vers une heuristique de la communication engageante}

$$
\text { (Bernard. 1998) présenté comporte plusieurs phases qui poursuivent des objectifs }
$$
complémentaires et qui sont inspirées par des présupposés associés.

En ce qui concerne les objectifs, il s'agit principalement de taire en sorte de renforcer les comportements de sécurité individuels effectifs et concrets sur le terrain au quotidien. Un tel objectif nécessite, dans certains cas de changer des comportements routiniers contre productifs en matière de sécurité, par exemple certaines « habitudes » concernant l'absence de port systématique de certains équipements de protection individuels (EPI) et notamment les lunettes ou les gants (le port du casque étant acquis dans tous les cas).

En ce qui concerne les présupposés, nous proposons de distinguer les présupposés suivants :

- les comportements de sécurité individuels de chaque agent sont étroitement associés à la mobilisation de toute la hiérarchie et de tous les personnels autour des enjeux sécurité. 
- la mobilisation s'inscrit dans des dynamiques de communication et plus spécifiquement de communication de projet.

- la sécurité ne peut reposer sur la logique « de la carotte et du bâton ». compliments, primes et médailles pour « la carotte » sanctions pour le «bâton ». dont on a mesuré les limites.

- la sécurité repose donc sur une responsabilisation des agents et la mise en œuvre d'actions précises et explicites : les agents prennent soin de leur sécurité individuelle pour « des raisons internes » et par des actes, non pas pour recevoir des récompenses matérielles ou symboliques ni pour éviter des sanctions.

En généralisant, un enjeu stratégique dans une organisation nécessite de penser et de mettre en place des actions de communication qui ont deux fonctions :

- premièrement, construire, montrer et valoriser la mobilisation de tous dans l'échange et dans une tension vers un but clairement défini (la sécurité)

- deuxièmement, mettre en lumière (c'est-à-dire en mots, textes, images et sons) les actions décidées et accomplies.

C'est une telle association de présupposés relevant de la communication ci de l'engagement qui structure le paradigme de la «communication engageante » auquel travaillent des équipes SIC et de psychologie sociale ${ }^{7}$

L'interdépendance de ces deux domaines, pensée et articulée de manière heuristique, est seule à pouvoir créer les conditions favorables d'une action individuelle et collective pertinente en termes d'objectifs, en l'occurrence plus de sécurité au travail pour chacun et pour tous, dans d'autres projets de recherche, il s'agit par exemple de plus de sécurité routière (Joule et Bernard. 2003).

Les phases du projet sont les suivantes:

- une phase «pied dans la porte » pour la hiérarchie qui consiste dans un processus communicationnel à informer, former et impliquer l'ensemble de la hiérarchie (direction, encadrement -ingénieurs, maître mineurs, agents de maîtrise), principalement sous la forme de journées de réflexion consacrées aux comportements sécurité où les maître mineurs réunissent les agents de maîtrise dont ils ont la responsabilité.

- une phase " pied dans la porte " pour les agents qui consiste dans un processus communicationnel sous lu forme également de journées de réflexion consacrées aux comportements sécurité réunissant un agent de maîtrise et les agents dont il a la responsabilité.

- une phase de communication transversale au cœur du dispositif car ces phases sont le lieu de construction du « soubassement comportemental engageant » et de la définition des actions mises en œuvre : elles prennent les formes de réunions, affiches, articles dans la presse interne notamment.

Lors des deux phases « pied dans la porte » les membres partagent leurs expériences de lu sécurité et définissent individuellement et collectivement des actions qu'ils décident de conduire afin d'améliorer leur sécurité concrètement au quotidien.

En fait, lors de ces journées de réflexion et d'un point de vue théorique, il s'agit moins d'échanger que d'effectuer un premier acte qui fonctionnera comme "soubassement comportemental préparatoire »: se déplacer du lieu de travail au lieu de lu formation, prendre lu parole publiquement, en se retrouvant autour d'une table dans un lieu qui n'est pas celui du travail quotidien pour débattre ce qui n'est pas une pratique habituelle pour des mineurs. De plus, l'identification de cet acte est bien rapportée au comportement sécurité. Il s'agit donc bien de la mise en place du «soubassement 
comportemental » dans lequel s'enracine la chaîne des actions sécurité qui suit in situ. Ce «soubassement comportemental «est engageant car il comporte certaines caractéristiques de l'engagement notamment les suivantes : il a un coût (déplacement, temps, etc.). un caractère public (prise de paroles face au groupe avec un membre de la hiérarchie de proximité), il est relativement « irréversible » (un suivi des actions décidées est mis en place).

La communication, non seulement traverse l'ensemble du projet ${ }^{8}$, mais elle est structurante car c'est dans les interactions, en réunion, qu'est construit le plan d'action. Les tonnes communicationnelles sont diversifiées: réunions inter-hiérarchiques, communication des actions et du suivi des actions (fiches de synthèse, affiche, articles dans la presse), communication du groupe de pilotage, etc. Lors de ces phases de communication inter-hiérarchiques. les thèmes qui préoccupent les agents, et qui sont des facteurs de stress donc à ce titre associés aux enjeux et pratiques de sécurité, par exemple la perspective «sombre » d'arrêter de travailler a 45 ans alors que les enfants dans de-nombreux cas sont encore en âge scolaire, que les épouses continueront à travailler, sont abordés. Il est apparu que le thème de la sécurité est interdépendant d'autres thèmes : identité', valorisation, appartenance à un collectif et sens au travail. On retrouve bien, cette fois-ci sur un plan " phénoménologique » l'interdépendance entre les questions du lien, du sens et de l'action. De ce point de vue la possibilité de verbaliser dans des petits groupes, ces autres thèmes d'une part a été perçue positivement par les agents et. d'autre part, d'un point de vue théorique, a permis de travailler la question de l'identification de l'acte en la tirant vers le haut, fin effet, et notamment, ce fut une occasion de mesurer que les préoccupations de chacun étaient dans de-nombreux cas celles de tous, mais que cependant, il y avait des modes d'approche et de résolution singuliers des problèmes qui pouvaient être modélisés et transférés afin de mieux vivre la période de deuil. Dans un tel contexte, celui de la mine et de la fermeture de la mine, le thème de la sécurité qui touche à ceux de la vie et de la mort est un thème qui est associé également à celui de la représentation de l'avenir. Ainsi, prendre soin de sa sécurité individuelle n'est donc plus seulement une question de conlormité aux règlements sécurité, voire, à un niveau d'identification de l'acte encore plus «bas» une gêne en supportant des EPI qui sont, dans certains cas « inconfortables » mais un enjeu vital qui engage l'avenir pour chacun et pour les proches (au travail, en famille et dans la cité1 ${ }^{10}$ ).

Enfin, le dispositif comporte la présence, dans les différentes phases, d'intervenants, chercheurs en psychologie sociale et en communication, qui veillent à ce que les initiatives et actions soient pertinentes, dans leur choix et leur conduite, avec le cadre de référence théorique défini, en l'occurrence engageantes pour plus de sécurité.

Sans entrer de manière plus précise dans le dispositif ni développer longuement l'analyse processuelle et les résultats, il convient d'indiquer que-la mobilisation obtenue, dans un cadre théorique rigoureux, a contribué à l'absence d'accidents et de blessures graves à un moment particulièrement délicat. Eneffet, la perspective de fermeture de la mine, pour des agents dont la moyenned'âge est de 45ans représente untournant à l'échelle de leur vie. Le travail de deuil (double deuil : de l'identité professionnelle et d'une tranche de v ie de 25uns en moyenne) et de redéfinition identitaire à accomplir (si je ne suis plus mineur, qui suis-je ?)est particulièrement important et pouvait conduire, pendant les derniers mois d'activité, à une baisse de vigilance et de motivation nuisible à la sécurité.

Enconclusion, le paradigme de "la communication engageante» qui propose de faire fonctionner, en les associant et enles articulant théoriquement et pratiquement, les 
enjeux du lien, du sens et de l'action, nous semble constituer une contribution à la problématique de l'«exister ensemble» dans les organisations. Le sens et le lien émergent de l'action dans l'interaction.

\section{BIBLIOGRAPHIE}

BERNARD. F Communication et engagement : ligures de rengagement paries actes. Actes du Colloque Communication et engagement. Nancy. Questions de Communication. 2003. (à paraître).

BERNARD. F., La communication de changement : vers une heuristique de l'induction. Communication et Organisation. GREC/O. Université Michel de Montaigne. Bordeaux. $\mathrm{n}^{\circ}$ 12. 1998. p. 302-337.

BERNARD. F.. Le management par projet : une logique de communication « imparfaite ». Communication et Organisation. GREC/O. Université Michel de Montaigne. Bordeaux, $\mathrm{n}^{\circ}$ 13. 1998. $\mathrm{p}$ 174-188.

BURGER. J.M.. The foot-in-the-door compliance procedure: a multiple-process analysis and review. Personality and Social Psychology Review, 3. 1999. p. 303-325.

CHANNOUF. A.. PY..J.. SOMAT. A.. Prédire des comportements à partir des altitudes : nouvelles perspectives in J.C. DESCHAMPS et. J.L BEAUVOIS (eds). La psychologie sociale. 2. Des attitudes aux attributions : sur la construction sociale de la réalité. Grenoble : Presses Universitaires de Grenoble. 1996. p. 55-65.

CHARTRAND. T.. PINCKERT. S.. BURGER. J.M.. When the manipulation Backfires: the effects of time delay and requester on the foot-in-the-door technique. Journal of Applied Social Psychology. 29. 1999. p. $211-221$.

JOULE. R.V., Le pied-dans-la-porte : un paradigme à la recherche d'une théorie. Psychologie Française. 32. 1987. p. 301-306.

JOULE R.V.. Quand les conduites résistent aux approches cognitives. In. J.L. BEAUVOIS. R.V. JOULE et J.M. MONTEIL. (eds.). Perspectives cognitives et conduites sociales. 3. Quelles Cognitions? Quelles Conduites ?Cousset : DelVal. 1989. p. 19-26.

JOULE. R.V.. La soumission librement consentie. In W. DOISE. N. DUBOIS. J.L. BEAUVOIS (eds.). La psychologie sociale 4. La construction sociale de la personne Grenoble: Presses Universitaires de Grenoble. 199. p. 233-246. JOULE R.V., Pour une communication organisationnelle engageante : vers un nouveau paradigme. Sciences de la société. 50 51.2000. p. 279-295.

JOULE. R.V. BEAUVOIS. J.L., La soumission librement consentie. Paris : Presses Universitaires de France. 1998.

JOULE. R.V. BEAUVOIS. J.L., Petit traité de manipulation à l'usage des honnêtes gens (nouvelle version), Grenoble : Presses Universitaires de Grenoble. 2002.

JOULE. R.V.. BERNARD. F.. Sécurité routière et engagement par les actes : pour de nouvelles pratiques de communication et de prévention. In P. Delhomme et F. Saad (eds). Psychologie de la conduite et sécurité routière. Paris: Edition Octares. 2003. 
KIESLER. C.A.. SAKUMURA. J.. A test of a model of commitment. Journal of Personality and Social Psychology. 3, 1966. p. 349-353.

LIPIANSKY. F.M.. Identité et communication. Paris : PUF. 1992.

\section{NOTES}

1. Sur ce point et le dossier « Management par projet et logiques communicationnelles » dans $C$ ommunication \& Organisation, 1998. $\mathrm{n}^{\circ} 13$

2. L'activité d'exploitation du charbon remonte au Moyen Age en Provence (14451. niais les plus vieux documents connus datent du XVII ${ }^{\mathrm{e}}$ siècle (site de Gardanne). Lors de la nationalisation. 4 sites étaient en activité : Gardanne. Gréasque. Meyreuil et Valdonne

3. Cf Rapport d'activité 2000.

4. L'histoire de la mine, en France et ailleurs, est ponctuée d accidents tragiques qui structurent, avec les contins sociaux, l'histoire de l'activité et de la mémoire ouvrière

5. Depuis sa présentation par Freedman et fraser en 1966. le paradigme du pied-dans-la-porte. n'a cessé de fasciner les chercheurs (cf Chartrand. Pinckert cl Hurger. 1999)

6. Les exemples cités sont issus des réunions organisées dans le programme sécurité présente

7. CREPCOM. Centre de recherches sur les pratiques de communication et de médiation et Laboratoire de Psychologie sociale de l'Université d'Aix-Marseille 1

8. Cf Bernard. 1998

9. Cf. le lien entre communication et identité, notamment tel qu'il a été étudié par Lipiansky (1992)

10. Il convient de rappeler la situation spécifique du logement des mineurs et de leurs familles Le " Statut du Mineur " accorde au personnel depuis 1946 le logement et le chauffage gratuits Autour des mines,se sont développées, souvent a la périphérie de communes rurales, les cites minières a l'architecture caractéristique le groupe Charbonnages de Francelierait, jusqu'en 1996 un parc de plus de plus de 110.000 logements La vie du mineur est donc caractérisée par une tonne de continuité entre le lieu de travail et le lieu de vie entre sphère professionnelle et sphère privée

\section{AUTEURS}

\section{FRANÇOISE BERNARD}

Université de Provence

ROBERT VINCENT JOULE

Université de Provence 